\title{
Nova espécie de Helicops Wagler, 1830 (Serpentes, Colubridae) do rio Tapajós, Amazônia, Brasil
}

\author{
Jossehan Galúcio da Frota \\ Laboratório de Herpetologia, Museu de Ciência e Tecnologia \& Faculdade de Biociências da Pontifícia \\ Universidade Católica do Rio Grande do Sul. Av. Ipiranga, 6681, 90619-900, Porto Alegre, RS, Brasil. E-mail: \\ jgfrota@hotmail.com.
}

\begin{abstract}
New species of Helicops Wagler, 1830 (Serpentes, Colubridae) from Tapajós river, Amazon region, Brazil. Within the 14 species of Helicops known, five of them are distributed in Brazilian Amazon region. Here I describe a new species of Helicops based on three specimens from Tapajós river, western Pará state, Brazil. The new species is characterized by an opaque, green-moss uniform coloration on the vertebral and paravertebral scales, extending until the end of the tail, becoming yellow-greenish in the paraventral region, without spots, blotches or stripes on the whole dorsum. It differs from all species known of Helicops by the dorsal color pattern. Morphologically, the new species seems like $H$. leopardinus (as indicated by the number of dorsal scale rows, the number of ventral scales, and the number of teeth), but it has smaller lobes in the hemipenis and a very distinct color pattern. Illustrations of the type material and a dicotomic key are presented to help to distinguish the new species from other species of Helicops from Brazilian Amazon region.
\end{abstract}

Keywords: Serpentes, Colubridae, Helicops tapajonicus sp. nov., northern Brazil Amazon region, Tapajós river.

Palavras-chave: Serpentes, Colubridae, Helicops tapajonicus sp. nov., norte do Brasil Amazônia, rio Tapajós.

\section{Introdução}

O gênero Helicops Wagler, 1830 é composto por 14 espécies, das quais $H$. angulatus (Linnaeus, 1758), H. danieli Amaral, 1938, H. hagmanni Roux, 1910, H. leopardinus (Schlegel, 1837), H. pastazae Shreve, 1934, H. petersi Rossman, 1976, H. polylepis Günther,

Recebido em 22 de setembro de 2004.

Aceito em 29 de setembro de 2005.

Distribuído em outubro de 2005.
1861, H. scalaris Jan, 1865, H. trivittatus (Gray, 1849) e H. yacu Rossman \& Dixon, 1975 ocorrem na Amazônia (Peters e OrejasMiranda 1970, Rossman 1970, 1975, 1976, 2002, Rossman e Dixon 1975, Yuki e Castano 1998). Cinco dessas espécies ocorrem no Brasil $(H$. angulatus, $H$. hagmanni, $H$. leopardinus, $H$. polylepis e $H$. trivittatus), sendo diferenciadas entre si pelo número de fileiras longitudinais de escamas dorsais, padrão de coloração dorsal e ventral e número de escamas subcaudais (Rossman 1970, 1973). 
No presente trabalho, é descrita uma nova espécie de Helicops com base em três espécimes coletados na região do baixo rio Tapajós, oeste do estado do Pará, Brasil.

\section{Material e Métodos}

Foram analisados 52 exemplares (Apêndice I), representando seis espécies de Helicops $(H$. angulatus, $H$. hagmanni, $H$. leopardinus, $H$. modestus, $H$. polylepis e $H$. trivittatus), depositados nas coleções do Museu de Ciências e Tecnologia da Pontifícia Universidade Católica do Rio Grande do Sul (MCP), do Museu Paraense Emílio Goeldi (MPEG) e do Laboratório de Pesquisas Zoológicas das Faculdades Integradas do Tapajós (FIT). Esses espécimes foram comparados com os três exemplares da nova espécie, depositados na coleção MCP.

Foram analisados caracteres merísticos, morfométricos, padrão de coloração e morfologia do hemipênis, esta conforme Dowling e Savage (1960). Os hemipênis do exemplar MCP 15382 foram preparados de acordo com os métodos descritos por Manzani e Abe (1988) e Pesantes (1994), com a técnica de coloração de Yuki (1997) e observados com auxílio de microscópico estereoscópico. As terminologias utilizadas para descrição dos caracteres corporais e hemipenianos seguem as propostas de Peters (1964) e Zaher (1999), respectivamente. A padronização da obtenção do número de escamas ventrais segue Dowling (1951), e a fórmula de redução de fileiras dorsais é apresentada segundo Thomas (1976).

Utilizaram-se as seguintes abreviaturas e siglas: CCA, comprimento da cabeça (medido desde a extremidade do rostral até a articulação posterior do quadrado-mandibular); CRC, comprimento rostro-cloacal; CC, comprimento da cauda. A altura e a largura da cabeça foram tomadas na região média das parietais. O comprimento do focinho foi medido da extremidade do rostral até a região anterior da narina. As medidas foram tomadas em milímetros $(\mathrm{mm})$ com o auxílio de fita métrica e paquímetro digital com precisão de 0,01 mm. O sinal “/” separa o lado direito (D) do esquerdo (E).

\section{Descrição da Espécie}

\section{Helicops tapajonicus sp. nov.} (Figuras 1-3)

Holótipo - MCP 15380, fêmea adulta, Comunidade de Parauá (02 50 '38” S, 55¹0’54” W), margem direita do rio Tapajós, município de Santarém, estado do Pará, Brasil. Coletado por João Lúcio em 20 de maio de 2001.

Parátipos - MCP 15381, fêmea adulta, e MCP 15382, macho adulto. Comunidade de Porto Novo (02³7’55”, $54^{\circ} 59^{\prime} 05^{\prime}$ 'W), margem esquerda do rio Tapajós, município de Belterra, estado do Pará, Brasil. Coletado por Frank Raynner V. Ribeiro em 25 de janeiro de 2003.

Diagnose - Helicops tapajonicus sp. nov. distingue-se das demais espécies de Helicops pela coloração dorsal, ventral e sub-caudal; coloração dorsal verde-musgo opaca uniforme nas regiões vertebral e paravertebral, sem manchas ou pintas ou estrias dorsais, tornandose amarelo-esverdeada nas regiões paraventrais; superfície ventral do corpo com manchas negras, que ocupam a metade lateral das escamas, alternando-se com manchas claras, resultando em um padrão xadrez, e, em determinadas partes do ventre, as manchas ocupam toda a largura da escama ventral, formando barras transversais que se estendem até a $2^{\text {a }}$ fileira de escamas dorsais; sub-caudais salpicadas irregularmente de negro. Além da coloração dorsal e ventral, $H$. tapajonicus sp. nov. diferencia-se de $H$. hagmanni, $H$. polylepis e $H$. trivittatus pelo número de fileiras longitudinais de escamas dorsais (Tabela 1); de H. angulatus, pelo número de escamas ventrais e escudos temporais, por não apresentar sub-caudais carenadas e na morfologia hemipeniana; e de $H$. leopardinus, pela disposição das manchas negras ventrais e que não se entendem além da $2^{\mathrm{a}}$ fileira de escamas dorsais e pela morfologia hemipeniana (Figura 4). 


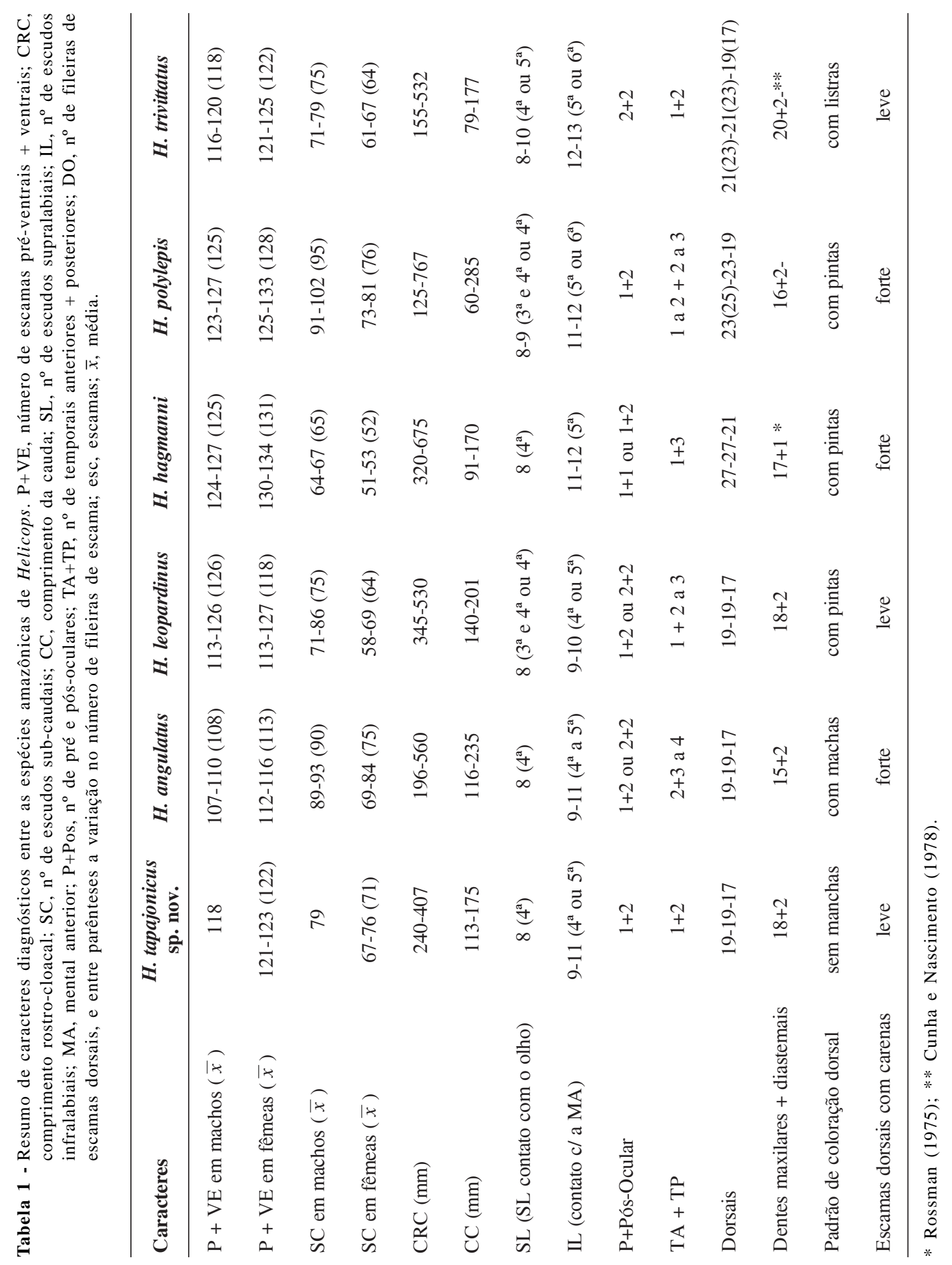

Phyllomedusa - 4(1), October 2005 


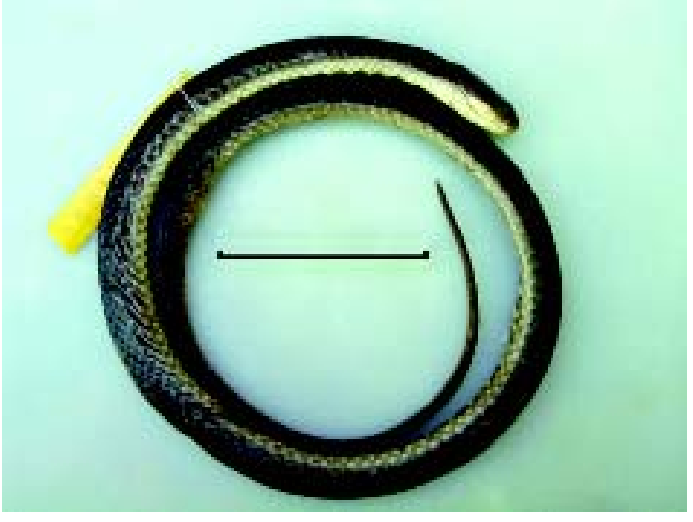

Figura 1 - Vista dorsal do holótipo (MCP 15380 \&) de Helicops tapajonicus sp. nov. Escala = $50 \mathrm{~mm}$.

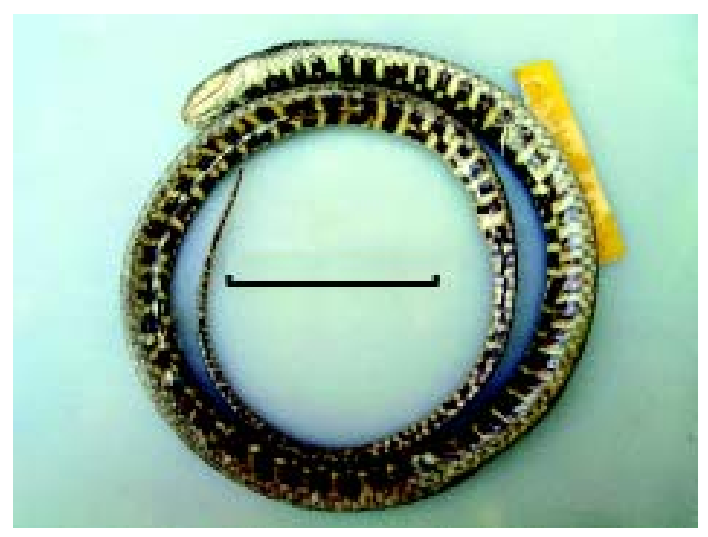

Figura 2 - Vista ventral do holótipo (MCP 15380 \&) de Helicops tapajonicus sp. nov. Escala $=$ $50 \mathrm{~mm}$.
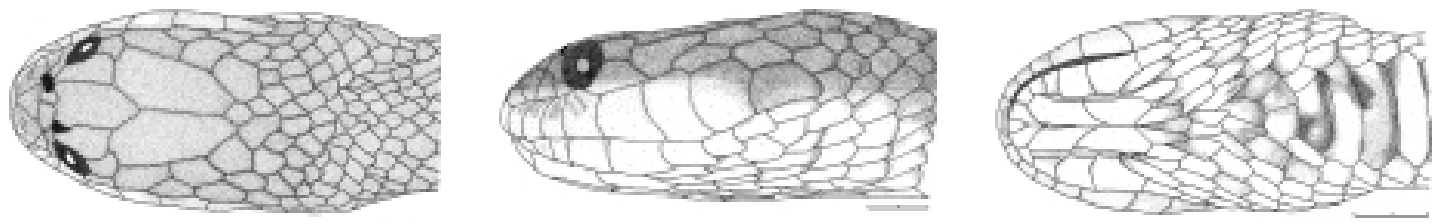

Figura 3 - Cabeça de Helicops tapajonicus sp. nov. (holótipo, MCP 15380 ㅇ) em vistas (A) dorsal, (B) lateral e (C) ventral. Escala $=5 \mathrm{~mm}$.
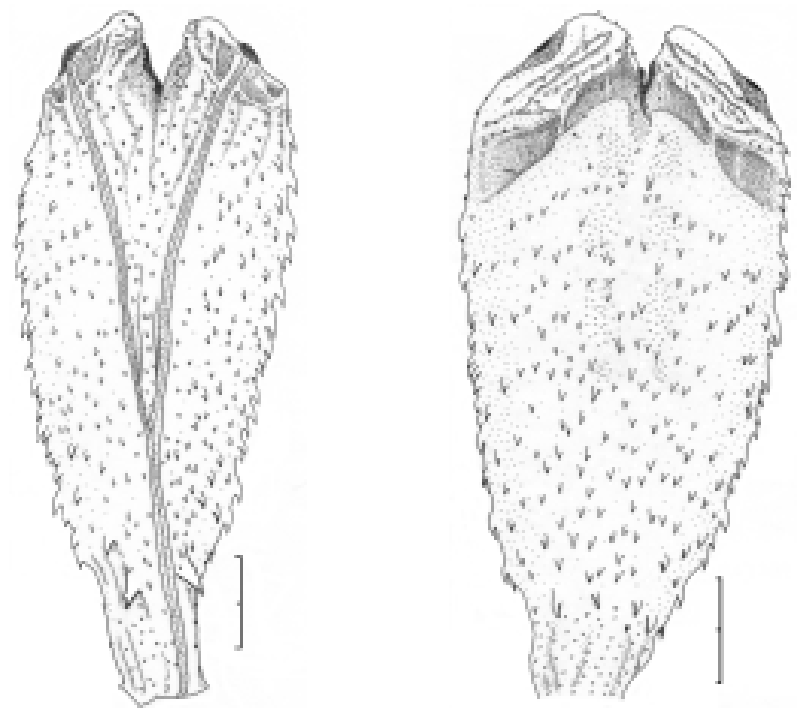

Figura 4 - Hemipênis direito de Helicops tapajonicus sp. nov. (MCP 15382); (A) face sulcada, (B) face assulcada. Escala $=2 \mathrm{~mm}$. 
Descrição do holótipo (Tabela 2) - Supralabiais $8 / 8$, a $4^{\mathrm{a}}$ em contato com a órbita ocular; infralabiais 9/10, 4/5 em contato com o primeiro par de escudos mentonianos; 1 pré-ocular e 2 pós-oculares; temporais 1+2; 1 pré-ventral, 121 ventrais; cloacal dividida; 76 pares de subcaudais; terminal cônica. Escamas dorsais dispostas em 19-19-17 fileiras longitudinais; fileira vertebral e paravertebrais levemente carenadas; paraventrais (cinco fileiras de escamas) lisas. Superfície da região mentoniana lisa, sem tubérculos e/ou granulações dérmicas. Escudo pré-frontal direito e porção anterior do supra-ocular esquerdo deformados. Dentição maxilar direita com 18 mais dois dentes diaste- mais; dentes maxilares sub-iguais, anteriores levemente menores do que os posteriores; dentes diastemais maiores que os dentes maxilares. Comprimento total $=582,0 \mathrm{~mm}(\mathrm{CRC}=407,0 \mathrm{~mm}$ e $\mathrm{CC}=175,0 \mathrm{~mm}$ ); e CC/CTO = 0,4; comprimento do focinho $=3,9 \mathrm{~mm}$, representando $16,7 \%$ do comprimento da cabeça; altura da cabeça $=8,5 \mathrm{~mm}$, representando $82,0 \%$ de sua largura $(10,4 \mathrm{~mm})$; olho com pupila redonda; diâmetro do olho $=2,36$ $\mathrm{mm}$; altura do rostral $=2,5 \mathrm{~mm}$, representando $61,0 \%$ da largura do rostral $(=4,1 \mathrm{~mm})$; largura do escudo loreal $=1,2 \mathrm{~mm}$, representando $64,0 \%$ de sua altura $(1,9 \mathrm{~mm})$; mental anterior levemente mais curta que a mental posterior (mental anterior/ mental posterior $=0,9 \mathrm{~mm}$ ).

Tabela 2 - Dados da folidose e morfometria da série-tipo de Helicops tapajonicus sp. nov. CRC, comprimento rostro-cloacal; CC, comprimento da cauda; CCA, comprimento da cabeça; P+VE, número de escamas pré-ventrais + ventrais; SC, $n^{0}$ de escamas sub-caudais; SL, $\mathrm{n}^{\circ}$ de escudos supralabiais (entre parênteses, $\mathrm{n}^{\circ}$ de SL em contato com a órbita ocular); IL, $\mathrm{n}^{\circ}$ escudos infralabiais (entre parênteses, $\mathrm{n}^{\circ}$ de $I L$ em contato com as mentais anteriores); DO, $\mathrm{n}^{\circ}$ de fileiras de escamas dorsais; CL, escama cloacal; $\bar{x}$, média; DP, desvio padrão.

\begin{tabular}{|c|c|c|c|c|}
\hline & Holótipo MCP 15380 & Parátipo MCP 15381 & Parátipo MCP 15382 & $\bar{x} \pm \mathrm{DP}$ \\
\hline Sexo & 우 & 우 & $\sigma^{x}$ & \\
\hline $\mathrm{CRC}(\mathrm{mm})$ & 407 & 400 & 240 & \\
\hline $\mathrm{CC}(\mathrm{mm})$ & 175 & 149 & 113 & \\
\hline $\mathrm{CCA}(\mathrm{mm})$ & 23,20 & 23,33 & 14,54 & \\
\hline $\mathrm{P}+\mathrm{VE}$ & $1+121$ & $2+123$ & $1+118$ & $120,6 \pm 2,5$ \\
\hline $\mathrm{SC}$ & 76 & 67 & 79 & $74 \pm 6,3$ \\
\hline SL & $8(4)$ & $8(4)$ & $8(4)$ & \\
\hline IL & $9(4) / 10(5)$ & $11(5)$ & $10(5)$ & \\
\hline $\mathrm{CL}$ & $1 / 1$ & $1 / 1$ & $1 / 1$ & \\
\hline Oculares & $1+2$ & $1+2$ & $1+2$ & \\
\hline Temporais & $1+2$ & $1+2$ & $1+2$ & \\
\hline Dentição & $18+2$ & $18+2$ & $18+2$ & \\
\hline DO & $19-19-17$ & $19-19-17$ & $19-19-17$ & \\
\hline Redução & $19(13) 19(63) \frac{3+4(91)}{3+4(93)} 17(112)$ & $19(12) 19(62) \frac{3+4(87)}{3+4(91)} 17(111)$ & $19(11) 19(62) \frac{4+5(93)}{4+5(90)} 17(102)$ & \\
\hline
\end{tabular}


Coloração do holótipo em vida (Figuras 1-3) Região dorsal da cabeça, tronco e cauda com coloração verde-musgo opaca, desde a cabeça até o ápice caudal; região paraventral amareloesverdeada. Coloração dos escudos rostral e supralabiais e da região gular amarelo-creme, com manchas escuras que não formam faixas transversais. Região ventral do tronco e cauda de cor laranja-avermelhada; manchas negras ocupam as metades laterais das escamas ventrais, atingindo levemente a primeira ou até a segunda fileira de escamas dorsais, resultando em um aspecto xadrez na maior parte da região ventral; algumas escamas ventrais totalmente manchadas de preto, formando estreitas barras transversais.

Coloração do holótipo preservado em álcool - Coloração dorsal da cabeça, tronco e cauda verde-musgo amarronzada, uniforme, tornandose pálida em toda a região paraventral. Escudos rostral, supralabiais e escamas gulares com coloração amarelo-esbranquiçada. Ventre amarelo-esbranquiçado com manchas escuras dispostas como descrito anteriormente (espécime em vida) (Figura 2).

Variação - Os dados referentes à folidose e às medidas dos parátipos estão sumarizados na Tabela 2. O exemplar MCP 15381 tem a coloração dorsal da cabeça verde-musgo opaca, entendendo-se por todo o escudo rostral e primeiras supralabiais; as manchas escuras sobre a região mediana das escamas pré-ventrais e ventrais não se estendem às escamas paraventrais. Diferenças entre machos e fêmeas foram encontradas no número de escamas ventrais, sub-caudais, no comprimento da cauda (Tabela 2) e na razão CC/ CRC (o 47\%, ㅇ 39-43\%).

Distribuição e hábitat - Helicops tapajonicus sp. nov., é conhecida apenas para as localidades de coleta do holótipo e parátipos. Os espécimes foram encontrados a noite, próximos da foz dos igarapés presentes em cada Comunidade, no rio Tapajós. No momento da coleta os espécimes estavam dentro d’água, em uma área lodosa com gramíneas, galhos caídos e raízes.
Hemipênis - A descrição a seguir baseia-se no hemipênis direito (Figura 4) do exemplar MCP 15382: bilobado, semicapitado, caliculado, com pequenos espinhos nos lóbulos; sulco espermático centrifugal bifurcando-se no meio do órgão; espinhos mais desenvolvidos lateralmente; espínulos na base, presentes nas duas faces do órgão. Face sulcada: região distal coberta com pequenos espinhos; região mediana e intra-sulcal com espinhos dispersos por todo o órgão. Face assulcada: região distal com duas séries de espinhos pequenos e com grandes cálices entre o corpo e os lóbulos do hemipênis; região mediana com espinhos dispersos, de tamanho mediano a grande, posicionados paralelamente ao sulco. O hemipênis alcança a $11^{\mathrm{a}}$ escama sub-caudal, quando invertido, apresentando $6,6 \%$ do comprimento rostro-cloacal e $14,0 \%$ do comprimento caudal do espécime. Cada lóbulo representa $20,8 \%$ do comprimento do órgão hemipeniano.

Etimologia - O epíteto específico "tapajonicus” é dado em referência ao rio Tapajós, no oeste do estado do Pará, localidade-tipo da nova espécie.

\section{Discussão}

A análise da distribuição geográfica mostra que $H$. tapajonicus sp. nov. apresenta distribuição alopátrica (diferentes bacias hidrográficas) em relação a $H$. danieli (Colômbia), $H$. pastazae (Peru, Equador, Colômbia e Venezuela), $H$. petersi (Equador), H. scalaris (Colômbia e Venezuela) e $H$. yacu (Peru), que ocorrem no extremo oeste da Amazônia (Rossman e Dixon 1975, Rossman 1976, 2002, Yuki 1994). No Brasil, a distribuição é alopátrica com relação a $H$. modestus Günther, 1861, que ocorre no cerrado (Nogueira 2001), e a H. carinicaudus (WiedNeuwied, 1825), H. gomesi Amaral, 1921 e $H$. infrataeniatus Jan, 1865, que ocorrem nas regiões sudeste e sul (Lema 2002, Aguiar e DiBernardo 2004). Helicops tapajonicus sp. nov. é simpátrica e sintópica com $H$. angulatus, $H$. 
leopardinus e $H$. polylepis, pois todas foram coletadas nos mesmos micro-hábtats nos municípios de Belterra e Santarém.

Helicops tapajonicus sp. nov. difere das outras espécies brasileiras amazônicas do gênero ( $H$. hagmanni, $H$. polylepis e $H$. Trivittatus) por apresentar um menor número de fileiras de escamas dorsais, além de outros caracteres (Tabela 1).

Helicops leopardinus é a espécie que mais se assemelha a $H$. tapajonicus sp. nov., compartilhando, entre outros caracteres, o número de fileiras de escamas dorsais (Tabela 1). O hemipênis de ambas é fracamente bilobado, semicapitado e possui espínulos na região distal e sulco espermático centrifugal; a região mediana tem espinhos dispersos, e a região proximal tem espinhos minúsculos, aparentando ser completamente nua. Difere, porém, além da coloração dorsal, ventral e sub-caudal, por apresentar o órgão hemipeniano mais largo e com lóbulos menores (20,8\% do comprimento do hemipênis). Para melhor distinção das espécies de Helicops da Amazônia brasileira, apresento a seguir uma chave dicotômica:

1. a) Mais de 19 fileiras de escamas dorsais no meio do corpo

b) 19 fileiras de escamas dorsais no meio do corpo

2. a) 23 ou menos fileiras de escamas dorsais no meio do corpo

b) Mais de 23 fileiras de escamas dorsais no meio do corpo hagmanni

3. a) Linhas claras longitudinais sobre o dorso; duas pré-oculares; ventre claro com duas fileiras longitudinais de pintas escuras trivittatus

b) Pintas escuras sobre o dorso; uma pré-ocular; região ventral escura com duas fileiras longitudinais de pintas claras..... polylepis
4. a) Sub-caudais carenadas lateralmente; amplas manchas transversais que cobrem todo o dorso estendendo-se até as escamas ventrais angulatus

b) Sub-caudais sem carenas; com ou sem pintas sobre o dorso. 5

5. a) Dorso com pintas; escamas ventrais com manchas negras transversais ultrapassando a $2^{\text {a }}$ fileira de escama paraventral; sub-caudais com manchas negras formando padrão barrado ou xadrezado leopardinus

b) Dorso sem pintas ou manchas; ventrais com ou sem manchas negras transversais que não ultrapassam a $2^{\text {a }}$ fileira de escama dorsais; sub-caudais salpicadas de negro. tapajonicus sp. nov.

\section{Agradecimentos}

Em especial a José Rodrigues da Frota, Dorotéia G. da Frota e César Aguiar, pelo apoio moral e financeiro; a Hipócrates de Menezes Chalkidis e Gina Synthia C. do Vale (FIT), Marcos Di Bernardo (MCT-PUCRS) e Ana Lúcia Prudente (MPEG) pelo empréstimo do material analisado. A Fernanda D’Agostini, Thales de Lema, Alfredo P. Santos-Jr e Luís Felipe Aguiar, pela leitura crítica e sugestões. A Lize Cappellari, Alessandra Travassos e Jorge S. Bernardo Silva, pela colaboração em diversas etapas deste trabalho. Este estudo foi parcialmente financiado pelo programa de Pós-Graduação da Coordenação de Aperfeiçoamento de Pessoal de Nível Superior (CAPES).

\section{Referências Bibliográficas}

Aguiar, L. F. S. e M. Di-Bernardo. 2004. Diet and feeding behavior Helicops infrataeniatus (Serpentes, Colubridae, Xenodontini) in Southern Brazil. Studies on Neotropical Fauna and Environment 39: $7-14$

Cunha, O. R. e F. R. Nascimento. 1978. Ofídios da Amazônia. X. As cobras da região leste do Pará. Publicações Avulsas do Museu Paranese Emílio Goeldi 31: 1-218. 
Dowling, H. G. 1951. A proposed standard system of counting ventral in snakes. British Journal of Herpetology 1: 97-99.

Dowling, H. G. e J. M. Savage. 1960. A guide to the snake hemipenis: a survey of basic structure and systematic characteristics. Zoologica 45: 17-28.

Lema, T. 2002. Os Répteis do Rio Grande do Sul: Atuais e Fósseis - Biogeografia - Ofidismo. Porto Alegre. EDIPUCRS. 264 pp.

Manzani, P. R. e A. S. Abe. 1988. Sobre dois novos métodos de preparo do hemipênis de serpentes. Memórias do Instituto Butantan 50: 15-20.

Nogueira, C. C. 2001. New records of Squamate reptiles in Central Brazilian Cerrado. II: Brasília Region. Hepetological Review 32: 285-287.

Pesantes, O. S. 1994. A method for preparing the hemipenis of preserved snakes. Journal of Herpetology 28: 93-95.

Peters, J. A. 1964. Dictionary of Herpetology. New York. Hafner Publishing Company. 392 pp.

Peters, J. A. e B. B. Orejas-Miranda. 1970. Catalogue of the Neotropical Squamata. Part I. Snakes. United States National Museum Bulletin 297: 1347.

Rossman, D. A. 1970. Helicops. in Peters, J. A. e B. Orejas-Miranda. Catalogue of the Neotropical Squamata. Part I. Snakes. United States National Museum Bulletin 297: 1-347.

Rossman, D. A. 1973. Miscellaneous notes on the South American water snake genus Helicops. HISS News-Journal 1: 189-191.

Rossman, D. A. 1975. Redescription of the South
American colubrid snake Helicops hagmanni Roux. Herpetologica 31: 414-418.

Rossman, D. A. 1976. Revision of the South American colubrid snakes of the Helicops pastazae complex. Occasional Papers of the Museum of Zoology, Louisiana State University 50: 1-15.

Rossman, D. A. 2002. Variation in the Xenodontid water snake Helicops scalaris Jan, and the status of $H$. hogei Lancini. Occasional Papers of the Museum of Zoology, Louisiana State University 78: $1-19$.

Rossman, D. A. e J. Dixon. 1975. A new colubrid snake of the genus Helicops from Peru. Herpetologica 31: 412-414.

Thomas, R. A. 1976. Dorsal scale row formulae in snakes. Copeia 1976: 839-841.

Yuki, R. N. 1994. Sobre Helicops danieli Amaral, 1937, com a descrição do hemipênis (Serpentes, Colubridae, Xenodontinae). Boletim do Museu Paraense Emílio Goeldi, Nova Série: Zoologia 10: 203-209.

Yuki, R. N. 1997. An observation technique for snake and lizard hemipenes with comments of preparation. Herpetological Review 28: 196.

Yuki, R. N. e V. Castano. 1998. Geographic distribution note of Water-snake Helicops danieli Amaral, 1937 (Colubridae: Xenodontinae). The Snake 28: 90-92.

Zaher, H. 1999. Hemipenial morphology of the South American xenodontine snakes, with a proposal for a monophyletic xenodontinae and a reappraisal of colubroid hemipenes. Bulletin of the American Museum of Natural History 214: 1-168 pp.

\section{Apêndice I - Material Examinado}

Helicops angulatus - ACRE: Porto Walter (MPEG 20381 +); AMAZONAS: Tabatinga (MCP 14106

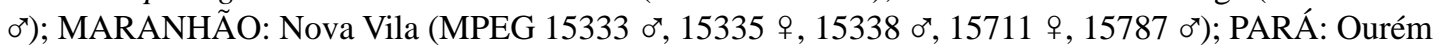
(MPEG 4993 ㅇ, 7002 ơ).

Helicops hagmanni - PARÁ: Capitão Poço (MPEG 10453 ㅇ, 10642 우, 13044 ơ, 14037 ㅇ), Ipitinga (MPEG 15513 ơ, 15515 ơ), São Domingos do Capim (MPEG 14480 우, 16331 ㅇ).

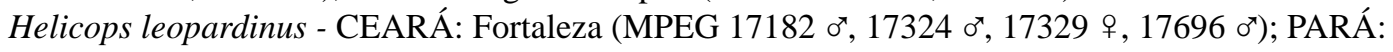
Almeirim (MPEG 16377 ㅇ) , Ilha do Marajós (MPEG 636 ㅇ, 638 ㅇ, 17717 ๙ 17718 ㅇ) , Melgaço (MPEG $199600^{\top}$ ); RONDÔNIA: Costa Marques (MCP $62580^{7}$ ).

Helicops modestus - SÃO PAULO: Cotia (MPEG 19204 ㅇ), Igaratá (MPEG 19201 우, $\left.19202 \sigma^{\top}\right)$.

Helicops polylepis - PARÁ: Belém (MPEG $1215 \sigma^{\top}$ ), Benevides (MPEG $8606 \sigma^{\star}$, $8619 \sigma^{7}$ ), Ilha do Marajó (MPEG 17720 ㅇ), Santarém (Taparí, LPHA 2251 ㅇ, 2618 ; Porto Novo LPHA 2455 ㅇ, 2458 ○), Viseu (MPEG 13902 ○’, 15099 우, 15105 ㅈ, 15112 ㅇ, 15120 ㅇ).

Helicops trivittatus - PARÁ: Belém (MPEG 1220 ㅇ) , Ilha do Marajó (MPEG 645 ox, 19629 우), Melgaço (MPEG 19971 ㅇ, 20482 ㅇ), Palestina do Pará (MPEG 12948 ox, 15545 ㅇ, 15546 ơ). 